\title{
Efficient reference-free adaptive artifact cancellers for impedance cardiography based remote health care monitoring systems
}

\author{
Madhavi Mallam ${ }^{1 *}$ and K. Chandra Bhushana Rao ${ }^{2}$
}

*Correspondence:
gurumadhu434@gmail.com
${ }^{1}$ Department of Electronics
and Communication
Engineering, Jawaharlal
Nehru Technological
University, Kakinada, AP
533003, India
Full list of author information
is available at the end of the
article

*Correspondence: gurumadhu434@gmail.com Department of Electronics and Communication Engineering, Jawaharla ru Technological 533003, India article

\begin{abstract}
In this paper, a new model for adaptive artifact cancelation in impedance cardiography (ICG) signals is presented. It is a hybrid model based on wavelet decomposition and an adaptive filter. A novel feature of this model is the implementation of referencefree adaptive artifact cancellers (AAC). For this implementation, the reference signal is constructed using a wavelet transformation. During critical conditions the filter weights may be negative and cause an imbalance in the convergence. To overcome this problem, we introduce non-negative adaptive algorithms in the proposed artifact canceller. To accelerate the performance of the AAC, we propose exponential non-negative and normalized non-negative algorithms to update the filter coefficients. The computational complexity of the filtering section in a remote health care system is important to avoid inter-symbol interference of the incoming samples. This can be achieved by combining sign-based algorithms with the adaptive filtering section. Finally, several AACs are developed using variants of the non-negative algorithms and performance measures are computed and compared. All of the proposed AACs are tested on actual ICG signals. Among the AACs evaluated, sign regressor normalized non-negative LMS $\left(S R N^{3} L M S\right)$ based adaptive artifact canceller achieves highest signal to noise ratio (SNR). The SNR achieved by this algorithm in baseline wander artifact elimination is $8.5312 \mathrm{dBs}$, in electrode muscle artifact elimination is $7.5908 \mathrm{dBs}$ and in impedance measurement artifact elimination is $8.4231 \mathrm{dBs}$.
\end{abstract}

Keywords: Artifact canceller, Cardiovascular diseases, Impedance cardiogram, Nonnegative algorithm, Remote health care

\section{Background}

Cardiovascular disease (CVD) refers to a large number of medical conditions relating to heart functionality. The World Health Organization (WHO) states that approximately $50 \%$ of all deaths from non-communicable diseases (NCDs) are from CVDs (World Health Organization 2015a). Among these, most of the deaths are outside the hospital because the patient is not treated in a timely manner. Additionally, the American Heart Association reported that more than 1 in 3 have more than one type of CVD (American Heart Association 2015) and that CVDs are the number 1 cause of death globally. In this scenario, WHO has planned to reduce the deaths from NCDs by $25 \%$ globally by 2025 (World Health Organization 2015b). Hence, research on cardiovascular health 
care technology is becoming intensely active. Among various methods of cardiac activity study, impedance cardiography (ICG) is a promising technique. ICG facilitates noninvasive and continuous monitoring of haemodynamic entities such as stroke volume $(\mathrm{SV})$ and cardiac output $(\mathrm{CO})$ in clinical scenarios. ICG measures the change in impedance that exists at the thorax from the physical activity of the heart muscle (Woltjer et al. 1997; Brown et al. 1994; Nagel et al. 1989). The methodologies and mathematical analyses used to calculate the ICG, SV and CO can be found in the literature (Scherhag et al. 2005; Kubicek et al. 1966; Sramek 1983; Kubicek et al. 1974; Bernstein 1986). Analysis of the ICG signal is an important task when treating a cardiac patient in critical conditions. However, during acquisition, the ICG signal encounters physiological and non-physiological artifacts. The artifacts include the baseline wander artifact (BWA), the electro-muscle artifact (EMA) and the impedance mismatch artifact (IMA). These artifacts cause changes in both the signal shape and tiny features, which are key parameters for clinical diagnoses. Therefore, to achieve high-resolution ICG signals for clinical investigations, the artifacts must be eliminated. Because most of the biomedical physiological and non-physiological phenomena are non-stationary, adaptive filtering techniques are likely to be a good remedy for this application. Adaptive filters can update their filter weights automatically to fit the input noise level. Several researchers have presented contributions on the analysis of ICG using signal processing techniques (Wang et al. 1995; Muzi et al. 1985; Ishiguro et al. 2006; Barros et al. 1995; Dromer et al. 2009; Yamamoto et al. 1988; Javaid et al. 2015; Sebastian et al. 2011). In these contributions, both least mean square (LMS) and recursive least square (RLS) algorithms are used. In a real-time clinical environment, and in critical conditions from an abnormal heart rhythm, the filter weights may be negative. The negative weights cause an imbalance in the convergence, resulting in poor filtering capability. To overcome this problem, we introduce non-negative adaptive algorithms in the proposed artifact canceller. To accelerate the performance of the AAC, we propose exponential non-negative and normalized non-negative algorithms to update the filter coefficients. The computational complexity of the filtering section in a remote health care system is important to avoid inter-symbol interference of the incoming samples. This can be achieved by combining sign-based algorithms with the adaptive filtering section. The remedy for unbalanced convergence and poor filtering performance of the algorithm is a modified LMS algorithm, in which a diagonal vector of the input is introduced in the weight update equation, i.e., a non-negative LMS $\left(N^{2} L M S\right)$ algorithm (Chen et al. 2011). This $N^{2} L M S$ keeps the filter weights from becoming negative from the abnormal rhythms of the heart. To improve the performance of the AAC, the $N^{2} L M S$ algorithm is varied, resulting in an exponential $N^{2} L M S\left(e N^{2} L M S\right)$ and a normalized $N^{2} L M S\left(N^{3} L M S\right)$ (Chen et al. 2014a, b). In conventional AACs, a reference signal, which is correlated with the noise component in the contaminated signal, is required (Thakor and Zhu 1991; Rahman et al. 2011; Karthik et al. 2013; Rahman et al. 2013). However, in a clinical environment, it is difficult to find a correlated reference. That is, the reference signal and the actual contaminated artifact in the ICG are not correlated. Therefore, a strategy using a discrete wavelet transformation (DWT) is implemented to construct a reference signal based on the contamination present in the actual signal (Peng et al. 2013). The AAC can then track the changes in the input signal, and, using wavelet decomposition methodology, 
automatically construct the reference signal. The reference signal is then utilized by the adaptive algorithm in the AAC to update the filter weight coefficients. In remote health care systems, computational complexity is also a factor that plays an important role when developing a lab on chip (LOC) or a system on chip (SOC) in modern health care telecardiology systems. Low complexity is desirable when constructing wearable and nano devices. In addition, if the computational complexity is large, the impulse response length of the receiver filters increases and thus the size of the filter increases. This cause inter-symbol interference at the input of the filter (Rahman et al. 2012). Therefore, to minimize computational complexity, and thus improving the suitability of the proposed AAC for remote health care systems, we combine the non-negative algorithms with the three simplified algorithms (Farhang-Boroujeny 1998). The simplified algorithms based on LMS recursion are known as sign regressor LMS (SRLMS), sign error LMS (SELMS) and sign sign LMS (SSLMS) algorithms. To reduce the computational complexity of the proposed algorithms, we combine the $e N^{2} L M S$ and the $N^{3} L M S$ algorithms with SRLMS, SELMS and SSLMS, resulting in the SReN $N^{2} L M S, S E e N^{2} L M S, S S e N^{2} L M S$, $S R N^{3} L M S, S E N^{3} L M S$ and $S S N^{3} L M S$ algorithms. Based on these algorithms, we use the wavelet decomposition method to develop several AACs to eliminate artifacts from ICG signals. Wavelet decomposition technique is particularly useful in health care applications; where accurate knowledge of the noise may not be available. The performance of these AACs is compared using the signal-to-noise ratio. The theory, the analysis of the algorithms and the simulation results of the various implementations are presented in the sections that follow.

\section{Methods}

In this paper, we introduce a new technique of artifact cancelation in ICG signals for remote health care monitoring systems. During signal acquisition in a typical ICG remote health care monitoring system, some physiological and non-physiological contaminants add to the actual heart activity, leading to ambiguous diagnoses and measurements. In addition to these contaminants, channel noise also masks the tiny features of the ICG signal. The major artifacts encountered with heart activity are the baseline wander artifact (BWA), the electro-muscle artifact (EMA) and the impedance mismatch artifact (IMA). The BWA is a base-line drift of the ICG signal from respiration activity. The EMA is caused by muscle activity, and the IMA is caused by an impedance mismatch between the electrodes and the skin, or from a mismatch of the electrodes. At the receiving end, a clear high-resolution signal is required to present to the doctor for diagnosis. In this context, AAC plays an important role. Figure 1 shows a block diagram of a wavelet-based AAC for remote health care monitoring systems.

The recorded ICG signal with artifact contaminants is expressed as follows:

$$
\operatorname{ICG}(n)=s(n)+n_{1}(n)
$$

where $I C G(n)$ is the recorded ICG signal; $s(n)$ is the original ICG signal generated from heart activity; and $n_{1}(n)$ is the artifact component (BWA or EMA or IMA or any combination of these three). In a remote system, $n_{1}(n)$ also includes channel noise.

The basic working principle of the proposed AAC is the following. The raw signal $\operatorname{ICG}(n)$ is input to the DWT decomposition unit. Using decomposition, a reference 


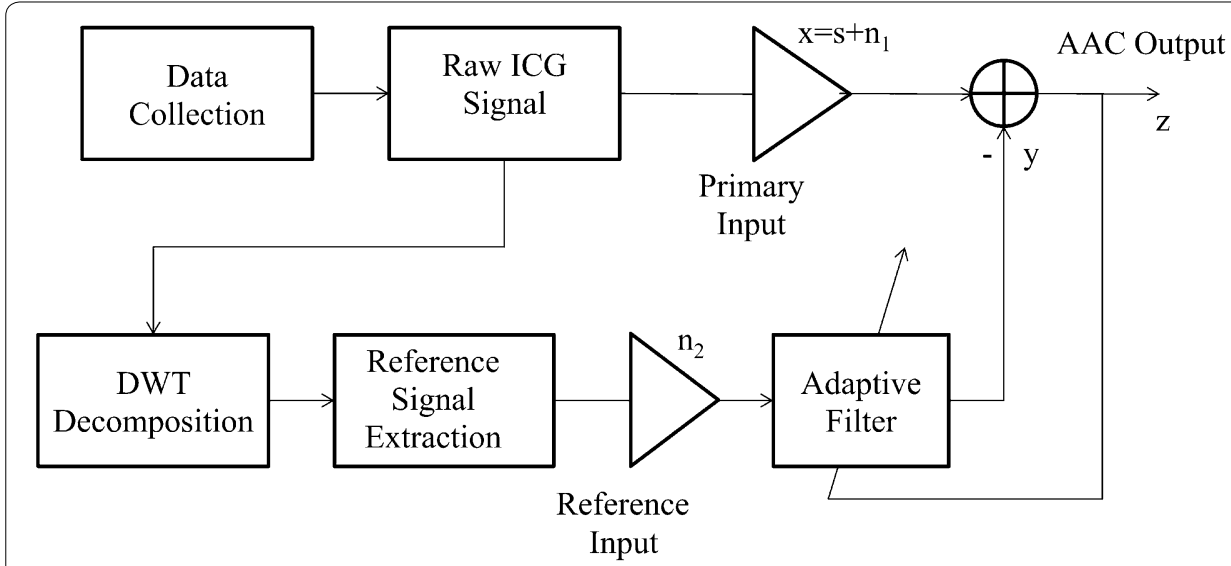

Fig. 1 Block diagram of wavelet-based adaptive artifact canceller for ICG signal analysis

signal is constructed for any type of contamination present in the raw input ICG signal. The constructed reference signal is used as the reference signal for the adaptive algorithm to update its weight coefficients. The proposed AAC thus plays a vital role in the implementation of an intelligent remote health care monitoring system that is referencefree by constructing the reference signal itself from the contaminated input signal.

\section{Construction of the reference signal from the noisy input signal}

The wavelet transform is used for signal decomposition in our model. It provides the temporal information for the signals whose frequency components are changing with time. The wavelet decomposition is a process of separating the signal into spectrally non-overlapping components. There are two categories of wavelet decomposition: continuous wavelet transforms (CWT) and discrete wavelet transforms (DWT). The CWT for a signal $s(n)$ is as follows:

$$
C W T(a, b)=\int_{-\infty}^{\infty} s(t) \frac{1}{\sqrt{a}} \varphi\left(\frac{t-b}{a}\right) d t
$$

where $a$ and $b$ are the scaling and shifting parameters, respectively, and $\varphi($.$) is the mother$ wavelet function. However, evaluating the scaling $(a)$ and shifting $(b)$ parameters for all possible scales is a computationally in feasible task. One possible way of solving the problem is choosing $a$ and $b$ as powers of two, in which case the DWT is as follows:

$$
D W T(a, b)=\frac{1}{\sqrt{2^{l}}} \int_{-\infty}^{\infty} s(t) \varphi\left(\frac{t-2^{l} m}{2^{l}}\right) d t
$$

where the scaling and shifting parameters are replaced by $2^{l}$ and $m 2^{l}$, respectively. Figure 2 shows the $L$-level wavelet decomposition of a signal $s(n)$. In this scheme, the signal ICG(n)first passes through the LP and HP filters, whose cut-off frequencies are one-fourth of the sampling frequency $f_{s}$ and down-sampled by 2, thus yielding an approximation $a_{1}$ and detail $d_{1}$, which are coefficients of the first level. The same procedure is employed on the first level of the approximation coefficients $a_{1}$, yielding the second level of approximation and detail coefficients. In this decomposition process, 


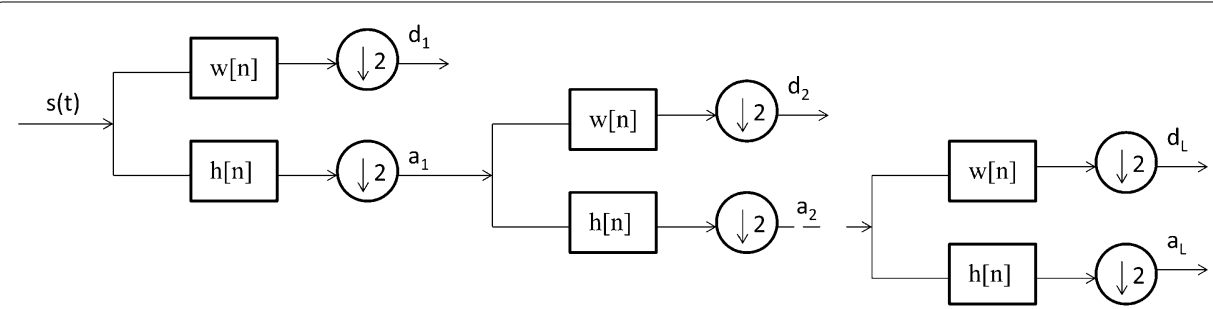

Fig. 2 L-level decomposition of an ICG signal

because of the down-sampling, the time resolution is halved and the frequency repulsion is doubled from the filtering operation. The frequency content of the signal at the $i$ th level decomposition is given by $0-f_{s} / 2^{i+1}$ and $0-f_{s} / 2^{i}, i=\{1,2, \ldots, L\}$ (Coifman and Donoho 1995; Percival and Walden 2000).

\section{Non-negative LMS-based algorithms for AACs}

In the proposed AAC, the input is the raw contaminated ICG signal and the reference is the signal constructed from the DWT decomposition of the raw ICG signal. This process is shown in Fig. 1. The AAC consists of an FIR filter of length $L$ taps. The weight coefficients are updated based on the weight update mechanism of various algorithms. The weight update mechanism for the basic LMS algorithm is as follows,

$$
\boldsymbol{W}(n+1)=\boldsymbol{W}(n)+\eta \boldsymbol{r}(n) e(n)
$$

where $W(n+1)$ is the next weight coefficient; $W(n)$ is the previous weight coefficient; $\eta$ is the step size; $r(n)$ is the reference signal, which is constructed from the DWT decomposition, required for training to eliminate noise from the raw signal $I C G(n)$; and $e(n)$ is the error generated, which is used as a feedback to the adaptive algorithm.

Because of the abnormalities in the ICG signal, i.e., the drastic variations in the signal features, the weight coefficients may become negative. This leads to poor performance of the adaptive algorithm in terms of convergence, stability and filtering capability. To overcome this drawback, a non-negative LMS $\left(N^{2} L M S\right)$ algorithm is proposed (Chen et al. 2011). The weight update mechanism is as follows:

$$
\boldsymbol{W}(n+1)=\boldsymbol{W}(n)+\eta \boldsymbol{D}(n) \boldsymbol{r}(n) e(n),
$$

where $D(n)$ is the diagonal matrix of the weight coefficients $W(n)$. The elaborated theory and analysis of $N^{2} L M S$ is presented by Chen et al. (2011).

In Eq. (4), each component of $W(n+1)$ is viewed as a variable step because of the combination of $\eta D(n)$. In the $N^{2} L M S$ algorithm, when the weights tend to zero, the convergence becomes unbalanced and the algorithm may diverge, causing the AAC to be ineffective for noise removal. To avoid the convergence imbalance characteristics in abnormal conditions, the exponential form $N^{2} L M S$ (e $\left.N^{2} L M S\right)$ is proposed. The weight update mechanism is then as follows:

$$
\mathbf{W}(n+1)=\mathbf{W}(n)+\eta \mathbf{r}(n) e(n) W^{\gamma}(n)
$$


For $0<\gamma<1$, the $n$th weight update in Eq. (5) is larger than that in Eq. (4), which accelerates convergence towards the steady state error. Another direct way to accelerate the convergence of $N^{2} L M S$ is normalization with respect to data. The normalized $N^{2} L M S$ $\left(N^{3} L M S\right)$ is mathematically expressed as follows:

$$
\mathbf{W}(n+1)=\mathbf{W}(n)+\eta(n) \mathbf{D}(n) \mathbf{r}(n) e(n)
$$

where $\eta(n)$ is a variable step size with respect to the reference input as follows:

$$
\eta(n)=\frac{\eta}{\alpha+r^{t}(n) r(n)}
$$

where $\alpha$ is a small constant used to avoid numerical difficulties. The elaborated theory and analysis of the $e N^{2} L M S$ and $N^{3} L M S$ algorithms are presented in the literature (Chen et al. 2014a, b).

To minimize the computational complexity of the above algorithms, and hence to make them suitable for remote health care applications, we combine the $e N^{2} L M S$ and $N^{3} L M S$ algorithms with the simplified algorithms described by Farhang-Boroujeny (Farhang-Boroujeny 1998). The weight update mechanism equations for the $e N^{2} L M S$ algorithm variants then become the following:

1. The sign regressor version of the $e N^{2} L M S$ algorithm uses the following weight update equation:

$$
\boldsymbol{W}(n+1)=\boldsymbol{W}(n)+\eta \operatorname{sign}(\boldsymbol{r}(n)) e(n) W^{\gamma}(n)
$$

This algorithm is the sign regressor $e N^{2} L M S\left(S R e N^{2} L M S\right)$ algorithm. The major advantage of this algorithm is its low computational complexity in terms of multiplications, independent of filter length. To compute Eq. (8), only one multiplication is required. Another important feature of the sign regressor (SR) algorithm is that its convergence characteristics are only slightly inferior to those of its normal version. This is caused by the normalization involved in the signum function (Farhang-Boroujeny 1998; Eweda 1990; Koike 1999).

2. The sign error version of the $e N^{2} L M S$ algorithm uses the following weight update equation:

$$
\boldsymbol{W}(n+1)=\boldsymbol{W}(n)+\eta \boldsymbol{r}(n) \operatorname{sign}(e(n)) W^{\gamma}(n)
$$

This algorithm is the sign error $e N^{2} L M S\left(S E e N^{2} L M S\right)$ algorithm.

3. The sign sign version of the $e N^{2} L M S$ algorithm uses the following weight update equation:

$$
\boldsymbol{W}(n+1)=W(n)+\eta \operatorname{sign}(\boldsymbol{r}(n)) \operatorname{sign}(e(n)) W^{\gamma}(n)
$$

This algorithm is the sign sign $e N^{2} L M S\left(S S e N^{2} L M S\right)$ algorithm. 
Similarly, the weight update mechanism equations for the $N^{3} L M S$ algorithm variants are written as follows:

1. The sign regressor version of the $N^{3} L M S$ algorithm uses the following weight update equation:

$\boldsymbol{W}(n+1)=\boldsymbol{W}(n)+\eta(n) \boldsymbol{D}(n) \operatorname{sign}(\boldsymbol{r}(n)) e(n)$

This algorithm is the sign regressor $N^{3} L M S\left(S R N^{3} L M S\right)$ algorithm.

2. The sign error version of the $N^{3} L M S$ algorithm uses the following weight update equation:

$\boldsymbol{W}(n+1)=\boldsymbol{W}(n)+\eta(n) \boldsymbol{D}(n) \boldsymbol{r}(n) \operatorname{sign}(e(n))$

This algorithm is the sign error $N^{3} L M S\left(S E N^{3} L M S\right)$ algorithm.

3. The sign sign version of the $N^{3} L M S$ algorithm uses the following weight update equation:

$$
\boldsymbol{W}(n+1)=\boldsymbol{W}(n)+\eta(n) \boldsymbol{D}(n) \operatorname{sign}(\boldsymbol{r}(n)) \operatorname{sign}(e(n))
$$

This algorithm is the sign sign $N^{3} L M S\left(S S N^{3} L M S\right)$ algorithm.

In Eqs. (11)-(13), during the normalization process, $r^{t}(n) r(n)$, in the denominator of $\eta(n)$, requires $L$ multiplications. To minimize the number of multiplications, we use only the maximum value of $r(n)$ instead of using all $L$ values. The new $\eta(n)$ is $\eta_{m}(n)$, as follows:

$$
\eta_{m}(n)=\frac{\eta}{\alpha+r_{m}^{t} r_{m}}
$$

The new weight update mechanisms for $N^{3} L M S$ and its three signed variants are then as follows:

$$
\begin{aligned}
& \mathbf{W}(n+1)=\mathbf{W}(n)+\eta_{m}(n) \mathbf{D}(n) \mathbf{r}(n) e(n) \\
& \mathbf{W}(n+1)=\mathbf{W}(n)+\eta_{m}(n) \mathbf{D}(n) \operatorname{sign}(\mathbf{r}(n)) e(n) \\
& \mathbf{W}(n+1)=\mathbf{W}(n)+\eta_{m}(n) \mathbf{D}(n) \operatorname{sign}(\mathbf{r}(n)) \operatorname{sign}(e(n)) \\
& \mathbf{W}(n+1)=\mathbf{W}(n)++\eta_{m}(n) \mathbf{D}(n) \operatorname{sign}(\mathbf{r}(n)) \operatorname{sign}(e(n))
\end{aligned}
$$

Figures 3 and 4 show the convergence curves of the $e N^{2} L M S$ and the $N^{3} L M S$ algorithms and their SR, SE and SS variants. The data in these figures show that $S R e N^{2} L M S$ is only slightly inferior to the $e N^{2} L M S$-based AAC, at the cost of a reduced number of multiplications. Hence, in practical implementations, if choosing between the SReN${ }^{2} L M S$ and the $e N^{2} L M S$ algorithms, the SR version is preferred. Similarly, between the $S R N^{3} L M S$ and the $N^{3} L M S$ algorithms, $S R N^{3} L M S$ is slightly inferior to $N^{3} L M S$, but uses a reduced number of multiplications. Therefore, for real-time applications, the $S R N^{3} L M S$ algorithm-based AAC can be used. $N^{3} L M S$ is slightly faster converging than $e N^{2} L M S$, as shown in Figs. 3 and 4. 


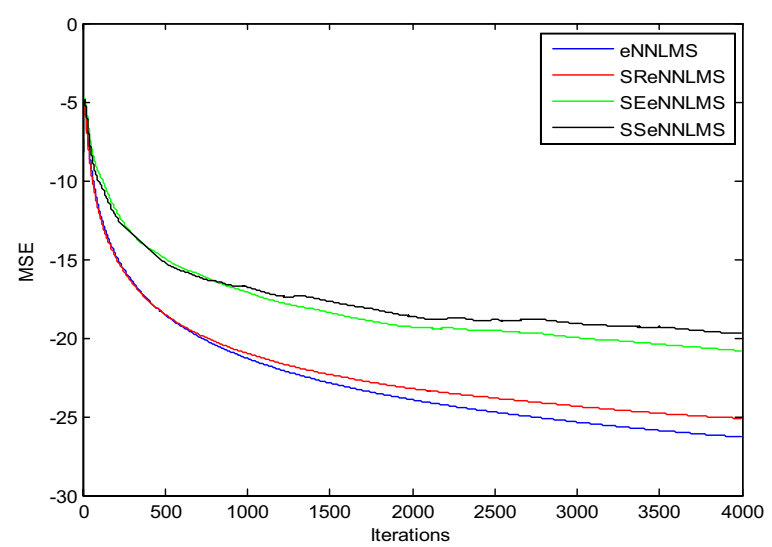

Fig. 3 Typical convergence curves for ICG signal enhancement in a Gaussian environment using exponential non-negative LMS (eN²LMS) and its variants

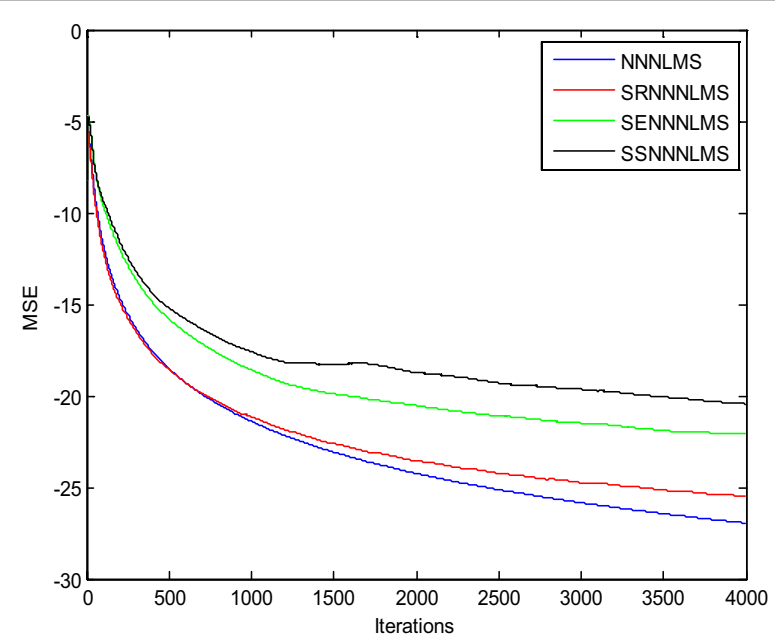

Fig. 4 Typical convergence curves for ICG signal enhancement in a Gaussian environment using normalized non-negative LMS ( $\left.N^{3} L M S\right)$ and its variants

\section{Results}

In our experiments, we use the ICG signals obtained from a VU-AMS ambulatory system (Goedhart et al. 2006; Riese et al. 2003; Willemsen et al. 1996). ICG signals with different artifacts are included in our simulations. We use five ICG signals with 5000 samples: Data1, Data2, Data3, Data4 and Data5. In our simulation results, we show 2000 samples to illustrate the high-resolution signals. To evaluate the performance of the algorithms discussed above, we develop various AACs using the LMS, $e N^{2} L M S$, $S R e N^{2} L M S$, SE eN $N^{2} L M S$, SS $e N^{2} L M S, N^{3} L M S$, SR $N^{3} L M S$, SE $N^{3} L M S$ and $S S N^{3} L M S$ algorithms. According to our proposed model, from the raw ICG signal, we construct a reference signal using DWT decomposition and use it as the reference in the adaptive algorithm. Using the above algorithms, we develop various AACs and calculate the signal-to-noise ratio (SNR), used as a measure of performance in our experiments. Comparisons of the SNR from the various algorithms are shown in Table 1. In addition to 
Table 1 Comparison of signal to noise ratio after ICG signal filtering due to various artifact cancelers (INDBS)

\begin{tabular}{|c|c|c|c|c|c|c|c|c|c|c|}
\hline $\begin{array}{l}\text { Artifact } \\
\text { type }\end{array}$ & $\begin{array}{l}\text { Sample } \\
\text { no. }\end{array}$ & LMS & $\begin{array}{l}\mathrm{eN}^{2} \\
\text { LMS }\end{array}$ & $\begin{array}{l}\text { SR } \\
e N^{2} \text { LMS }\end{array}$ & $\begin{array}{l}\text { SE } \\
e N^{2} L M S\end{array}$ & $\begin{array}{l}\text { SS } \\
\text { eN } N^{2} \text { LMS }\end{array}$ & $N^{3}$ LMS & $\begin{array}{l}\text { SR } \\
N^{3} \text { LMS }\end{array}$ & $\begin{array}{l}\text { SE } \\
N^{3} \text { LMS }\end{array}$ & $\begin{array}{l}\text { SS } \\
N^{3} L M S\end{array}$ \\
\hline \multirow[t]{6}{*}{ BWA } & Data 1 & 4.9815 & 5.8286 & 7.1277 & 5.2723 & 5.0801 & 8.3075 & 8.4956 & 5.7226 & 5.2454 \\
\hline & Data 2 & 4.9783 & 5.8251 & 7.1626 & 5.2583 & 5.1438 & 8.3059 & 8.5461 & 5.6331 & 5.3726 \\
\hline & Data 3 & 4.9876 & 5.8359 & 7.1035 & 5.2348 & 4.9496 & 8.3081 & 8.4711 & 5.5724 & 5.3241 \\
\hline & Data 4 & 4.9993 & 5.8307 & 7.1964 & 5.2934 & 5.1364 & 8.3317 & 8.5839 & 5.6587 & 5.4356 \\
\hline & Data 5 & 4.9782 & 5.8289 & 7.1732 & 5.1804 & 5.1423 & 8.3184 & 8.5595 & 5.7144 & 5.3976 \\
\hline & Average & 4.1884 & 5.8298 & 7.1531 & 5.2480 & 5.0904 & 8.3143 & 8.5312 & 5.5502 & 5.3551 \\
\hline \multirow[t]{6}{*}{ EMA } & Data 1 & 4.3813 & 3.9164 & 6.8491 & 3.0812 & 3.0628 & 7.4958 & 7.5570 & 4.8956 & 4.7859 \\
\hline & Data 2 & 4.3905 & 3.9213 & 6.9333 & 3.1025 & 3.0994 & 7.4984 & 7.5823 & 4.8425 & 4.6926 \\
\hline & Data 3 & 4.3802 & 3.9172 & 6.7805 & 3.1583 & 3.1133 & 7.4884 & 7.6118 & 4.8520 & 4.7085 \\
\hline & Data 4 & 4.4016 & 3.2729 & 6.8429 & 3.1693 & 3.1501 & 7.5071 & 7.5523 & 4.9425 & 4.6538 \\
\hline & Data 5 & 4.3912 & 3.9256 & 7.1351 & 3.1979 & 3.1612 & 7.4979 & 7.6445 & 4.8078 & 4.6705 \\
\hline & Average & 4.3889 & 3.9216 & 6.9081 & 3.1418 & 3.1173 & 7.5268 & 7.5908 & 4.8701 & 4.7022 \\
\hline \multirow[t]{6}{*}{ IMA } & Data 1 & 4.0359 & 3.8136 & 7.5765 & 6.2971 & 4.5841 & 7.1845 & 8.4466 & 7.8773 & 5.2710 \\
\hline & Data 2 & 4.0256 & 3.8371 & 7.5896 & 6.2309 & 4.6642 & 7.1732 & 8.4371 & 7.8473 & 5.1835 \\
\hline & Data 3 & 3.9916 & 3.8096 & 7.5669 & 6.2985 & 4.7363 & 7.1686 & 8.4461 & 7.8435 & 5.1477 \\
\hline & Data 4 & 3.9912 & 3.8140 & 7.5677 & 6.2338 & 4.7059 & 7.1854 & 8.4461 & 7.8435 & 5.1477 \\
\hline & Data 5 & 3.9939 & 3.8079 & 7.5611 & 6.2145 & 4.6768 & 7.1713 & 8.3606 & 7.8658 & 5.2168 \\
\hline & Average & 4.0076 & 3.8164 & 7.5723 & 6.2551 & 4.6633 & 7.1766 & 8.4231 & 7.8578 & 5.1884 \\
\hline
\end{tabular}

SNR measurements, we also tabulated the weight coefficients used to enhance Data1 during various artifact cancelations to examine the non-negative constraints of the nonnegative algorithms, as shown in Table 2. The data in Table 2 show that the non-negative algorithms keep the weights from becoming negative. In our simulations, the filter length is 10 and the step size is 0.1 . Because of space constraints, only the simulation results from Data1 are shown in this paper. ICG signals contaminated with BWA, EMA and IMA are used to illustrate the enhancement process.

\section{Discussion}

\section{Baseline-wander artifact (BWA) removal using the DWT adaptive artifact canceller}

This experiment demonstrates the baseline wander artifact cancelation from the ICG signal. The raw ICG signal is input to the DWT-based AAC, as shown in Fig. 1. Using decomposition, DWT constructs a reference signal. This signal is effectively used as a reference signal to the AAC, as shown in the block diagram as $n_{2}$. Using feedback from the output $z(n)$, the algorithm trains $n_{2}$ to closely correlate with the artifact component $n_{1}$ in the input ICG signal $x(n)$. The SNR and the relative root mean square error (RRMSE) were used to measure the performance of the DWT-based AAC for BWA cancelation. The filtering results of the BWA cancelation are shown in Fig. 5. The results shown in Fig. 5d, g, h are clearer than the results from the other AACs. Figure 6 shows the residual error component after filtering with the various algorithms. The data in Fig. 6h show that the residual error in the case of $S R N^{3} L M S$ is less than that of the other algorithms. This is also supported by the SNR table, i.e., from among all of the algorithms, $S R N^{3} L M S$ achieves the highest SNR, $8.4956 \mathrm{dBs}$. The data in Table 2 also show that all of the non-negative algorithms have non-negative weight coefficients when 
Table 2 Comparison of weight coefficient variations due to various artifact cancelers for data 1

\begin{tabular}{|c|c|c|c|c|c|c|c|c|c|c|c|}
\hline Artifact type & Algorithm & W1 & W2 & W3 & W4 & W5 & W6 & W7 & W8 & W9 & W10 \\
\hline \multirow[t]{9}{*}{ BWA } & LMS & 0.0559 & 0.0625 & 0.0692 & 0.0759 & 0.0828 & 0.0896 & 0.0965 & 0.1033 & 0.1102 & 0.1170 \\
\hline & eN²LMS & 0.0792 & 0.0804 & 0.0817 & 0.0830 & 0.0843 & 0.0857 & 0.0870 & 0.0884 & 0.0898 & 0.0912 \\
\hline & SReN $N^{2} L M S$ & 0.0435 & 0.0467 & 0.0505 & 0.0560 & 0.0618 & 0.0703 & 0.0805 & 0.0918 & 0.1026 & 0.1177 \\
\hline & SEeN $N^{2} L M S$ & 0.0810 & 0.0835 & 0.0860 & 0.0885 & 0.0911 & 0.0936 & 0.0962 & 0.0988 & 0.1013 & 0.1039 \\
\hline & SSeN ${ }^{2} L M S$ & 0.0636 & 0.0670 & 0.0705 & 0.0742 & 0.0780 & 0.0820 & 0.0861 & 0.0904 & 0.0949 & 0.0995 \\
\hline & $N^{3} L M S$ & 0.0606 & 0.0623 & 0.0641 & 0.0660 & 0.0679 & 0.0699 & 0.0719 & 0.0740 & 0.0761 & 0.0783 \\
\hline & SRN ${ }^{3} \mathrm{LMS}$ & 0.0604 & 0.0633 & 0.0664 & 0.0699 & 0.0734 & 0.0780 & 0.0829 & 0.0882 & 0.0929 & 0.0987 \\
\hline & SEN $N^{3} L M S$ & 0.0788 & 0.0805 & 0.0821 & 0.0838 & 0.0855 & 0.0872 & 0.0890 & 0.0907 & 0.0924 & 0.0941 \\
\hline & SSN ${ }^{3} L M S$ & 0.0694 & 0.0717 & 0.0742 & 0.0767 & 0.0793 & 0.0820 & 0.0848 & 0.0876 & 0.0906 & 0.0937 \\
\hline \multirow[t]{9}{*}{ EMA } & LMS & 0.1516 & 0.1338 & 0.1218 & 0.1162 & 0.1162 & 0.1222 & 0.1335 & 0.1497 & 0.1704 & 0.1953 \\
\hline & eN²LMS & 0.1040 & 0.1007 & 0.0984 & 0.0971 & 0.0967 & 0.0973 & 0.0987 & 0.1010 & 0.1040 & 0.1079 \\
\hline & SReN $N^{2} L M S$ & 0.2284 & 0.2284 & 0.2284 & 0.2284 & 0.2284 & 0.2284 & 0.2284 & 0.2284 & 0.2247 & 0.4283 \\
\hline & SEeN $N^{2}$ LMS & 0.1364 & 0.1307 & 0.1251 & 0.1296 & 0.1342 & 0.1390 & 0.1439 & 0.1490 & 0.1543 & 0.1596 \\
\hline & SSeN ${ }^{2} L M S$ & 0.1166 & 0.1127 & 0.1090 & 0.1134 & 0.1179 & 0.1225 & 0.1273 & 0.1321 & 0.1371 & 0.1422 \\
\hline & $N^{3} L M S$ & 0.1052 & 0.0979 & 0.0939 & 0.0931 & 0.0950 & 0.0996 & 0.1067 & 0.1163 & 0.1283 & 0.1429 \\
\hline & $S R N^{3} L M S$ & 0.1013 & 0.0877 & 0.0797 & 0.0713 & 0.0705 & 0.0689 & 0.0945 & 0.1087 & 0.1112 & 0.1276 \\
\hline & SEN ${ }^{3} L M S$ & 0.0646 & 0.0501 & 0.0387 & 0.0501 & 0.0672 & 0.0886 & 0.1167 & 0.1540 & 0.2034 & 0.2689 \\
\hline & $S S N^{3} L M S$ & 0.1447 & 0.1304 & 0.1175 & 0.1323 & 0.0953 & 0.1072 & 0.1206 & 0.1357 & 0.1527 & 0.1717 \\
\hline \multirow[t]{9}{*}{ IMA } & LMS & 0.1459 & 0.1498 & 0.1538 & 0.1580 & 0.1622 & 0.1665 & 0.1708 & 0.1752 & 0.1795 & 0.1839 \\
\hline & eN $N^{2} L M S$ & 0.0979 & 0.0989 & 0.0999 & 0.1010 & 0.1021 & 0.1033 & 0.1044 & 0.1056 & 0.1068 & 0.1080 \\
\hline & SReN $N^{2} L M S$ & 0.0956 & 0.0964 & 0.0978 & 0.0987 & 0.1009 & 0.1014 & 0.1023 & 0.1035 & 0.1041 & 0.1064 \\
\hline & SEeN $N^{2}$ LMS & 0.0530 & 0.0543 & 0.0556 & 0.0570 & 0.0584 & 0.0598 & 0.0613 & 0.0629 & 0.0644 & 0.0660 \\
\hline & SSeN $N^{2} L M S$ & 0.0474 & 0.0540 & 0.0612 & 0.0693 & 0.0781 & 0.0838 & 0.0985 & 0.1101 & 0.1229 & 0.1368 \\
\hline & $N^{3} L M S$ & 0.1034 & 0.1054 & 0.1074 & 0.1095 & 0.1116 & 0.1138 & 0.1160 & 0.1183 & 0.1205 & 0.1229 \\
\hline & SRN ${ }^{3} L M S$ & 0.0381 & 0.0451 & 0.0553 & 0.0674 & 0.0847 & 0.1081 & 0.1374 & 0.1779 & 0.2322 & 0.3072 \\
\hline & SEN $N^{3} L M S$ & 0.0087 & 0.0090 & 0.0094 & 0.0098 & 0.0102 & 0.0106 & 0.0110 & 0.0114 & 0.0118 & 0.0121 \\
\hline & $S S N^{3} L M S$ & 0.1347 & 0.4287 & 0.2743 & 0.3513 & 0.4125 & 0.3187 & 0.8231 & 0.5349 & 0.1250 & 0.4781 \\
\hline
\end{tabular}

filtering various artifacts. Figures 7 and 8 illustrate the RRMSE (in \%) calculated during BWA cancelation. The data in Figs. 7 and 8 show that $e N^{2} L M S, N^{3} L M S$ and their sign regressor versions perform better the other versions. These algorithms achieve the minimum residual error from among all of the tested algorithms. Finally, from all of the performance measures, $S R N^{3} L M S$ is better than the other algorithms with respect to SNR, RRMSE, convergence and computational complexity.

\section{Electro-muscle artifact (EMA) removal using the DWT adaptive artifact canceller}

This experiment demonstrates electro-muscle artifact cancelation from an ICG signal. The raw ICG signal is input to the DWT-based AAC, as shown in Fig. 1. The DWT constructs a reference signal using decomposition. This signal is effectively used as a reference signal to the AAC, as shown in the block diagram as $n_{2}$. Using feedback from the output $z(n)$, the algorithm trains $n_{2}$ to closely correlate it with the artifact component $n_{1}$ in the ICG input signal $x(n)$. The SNR is used to measure the performance of the DWTbased AAC for EMA cancelation. The filtering results of the EMA cancelation are shown 

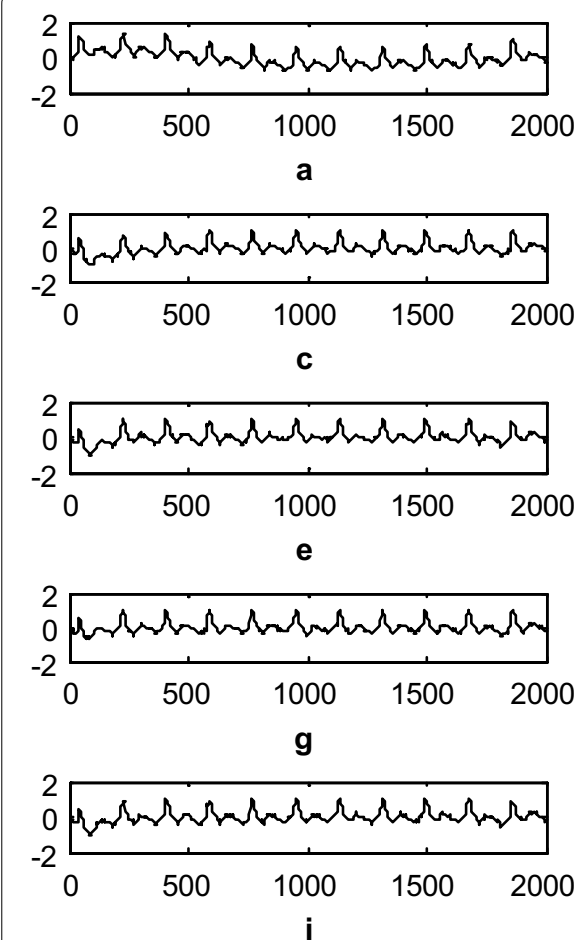
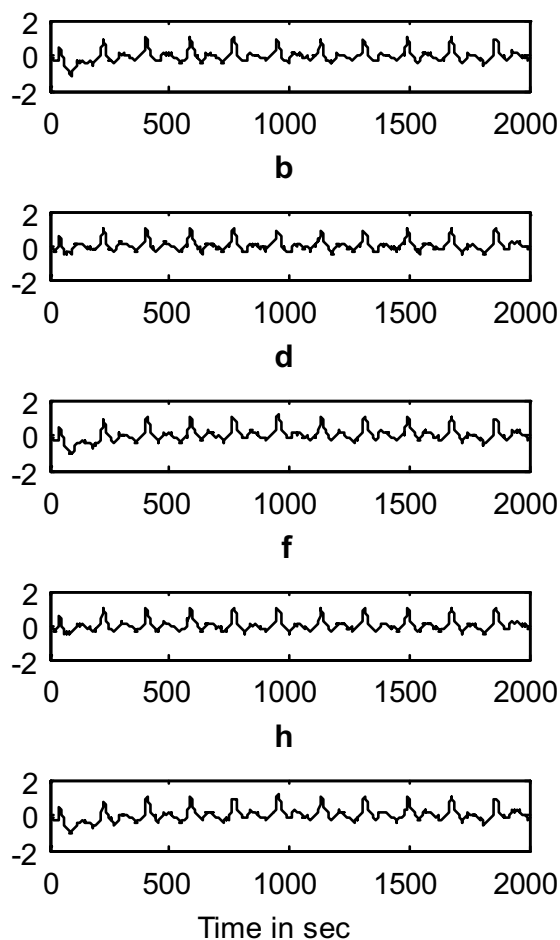

Fig. 5 Results of filtering BWA using various AACs (a). ICG signal with BWA (b). ICG filtering with LMSupdated AAC (c). ICG filtering with exponential non-negative LMS (eN² LMS)-updated AAC (d). ICG filtering with SReN²LMS-updated AAC (e). ICG filtering with SEeN ${ }^{2} L M S$-updated AAC (f). ICG filtering with SSeN ${ }^{2} L M S-$ updated AAC (g). ICG filtering with normalized non-negative LMS ( $N^{3} L M S$ )-updated AAC (h). ICG filtering with SRN ${ }^{3} L M S-u p d a t e d$ AAC (i). ICG filtering with SEN ${ }^{3} L M S$-updated AAC, and (j). ICG filtering with SSN ${ }^{3} L M S-$ updated AAC. (The $x$-axis is the number of samples and the $y$-axis is the signal amplitude in $\mathrm{mV}$ )

in Fig. 9. The results shown in Fig. 9d, g, h are clearer than the results from the other AACs. Figure 10 shows the residual error component after filtering with various algorithms. The data in Fig. 10h show that the residual error in the case of $S R N^{3} L M S$ is less than that for the other algorithms. This conclusion is supported by the SNR table; among all of the algorithms $S R N^{3} L M S$ achieves the highest SNR of $7.5570 \mathrm{dBs}$. The data in Table 2 also show that all of the non-negative algorithms use non-negative weight coefficients when filtering various artifacts. Finally, to summarize the performance measures, is the results show that $S R N^{3} L M S$ is better than the other algorithms with respect to SNR, RRMSE, convergence and computational complexity.

\section{Impedance mismatch artifact (IMA) removal using the DWT adaptive artifact canceller}

This experiment demonstrates impedance mismatch artifact cancelation from the ICG signal. The raw ICG signal is input to the DWT-based AAC, as shown in Fig. 1. The DWT constructs a reference signal using decomposition. This signal is effectively used as a reference signal for the AAC, as shown in the block diagram as $n_{2}$. Using feedback from the output $z(n)$, the algorithm trains $n_{2}$ to closely correlate with the artifact component $n_{1}$ in the input ICG signal $x(n)$. The SNR is used to measure the performance of 

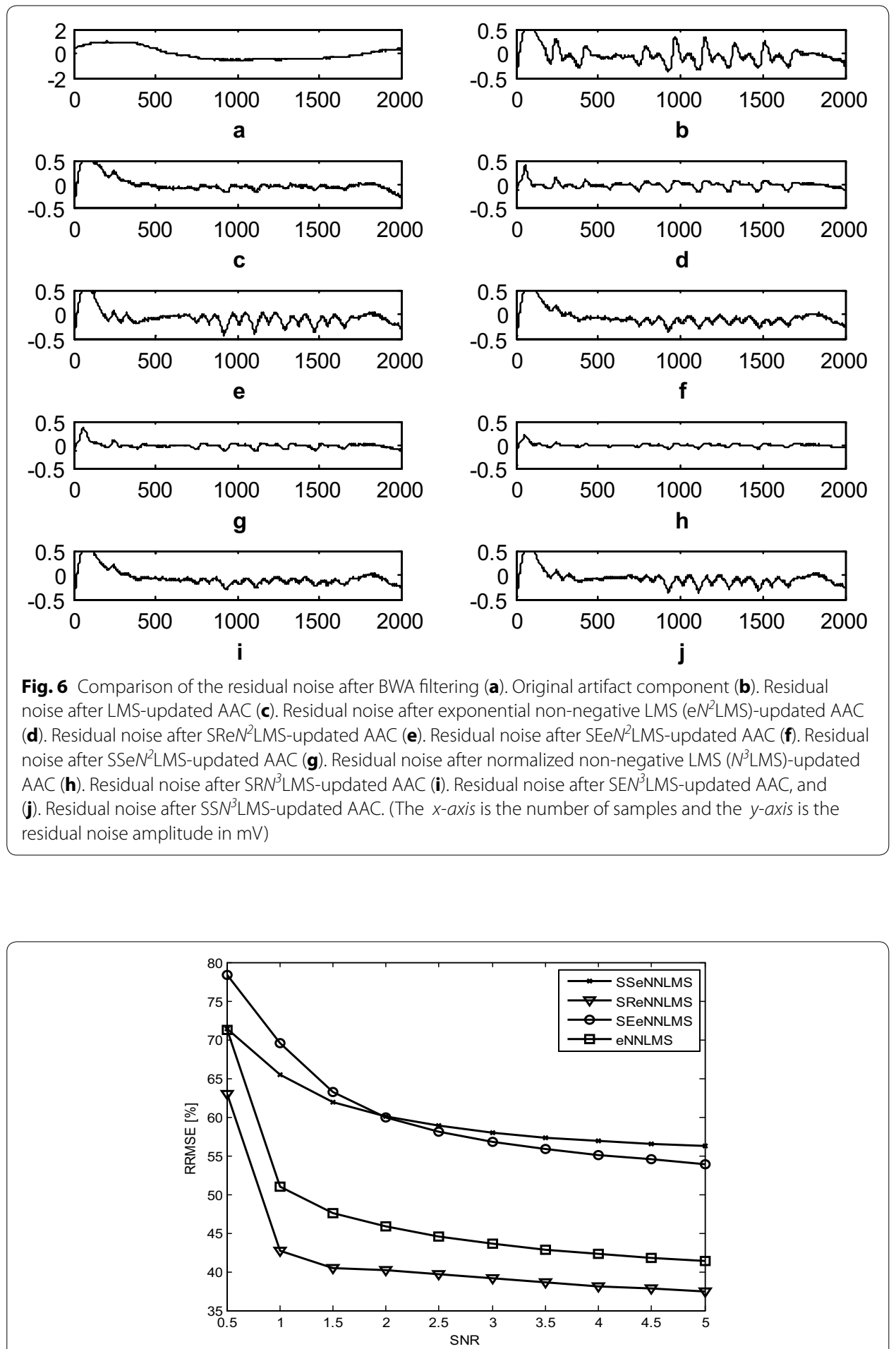

Fig. 7 Comparison of the RRMSE of exponential non-negative LMS and its signed versions

the DWT-based AAC for EMA cancelation. The filtering results of the EMA cancelation are shown in Fig. 11. The results shown in Fig. 11d, g, h are clearer than the results for the other AACs. Figure 12 shows the residual error component after filtering with the 


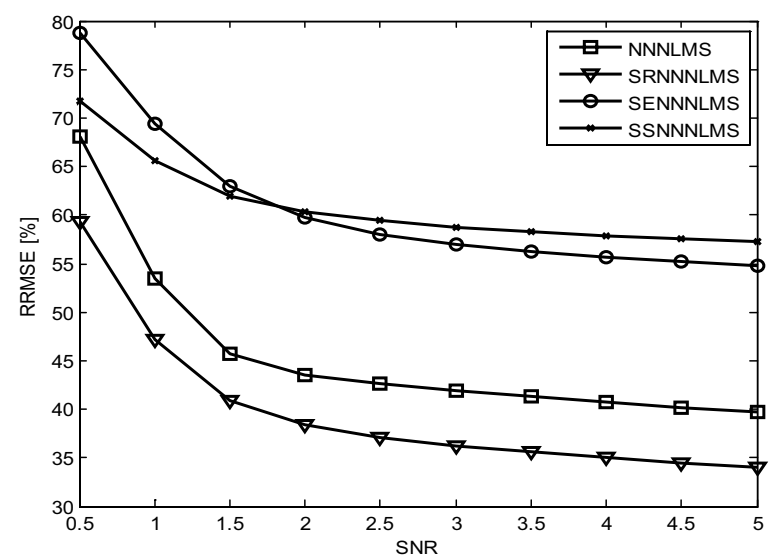

Fig. 8 Comparison of the RRMSE of normalized non-negative LMS and its signed versions

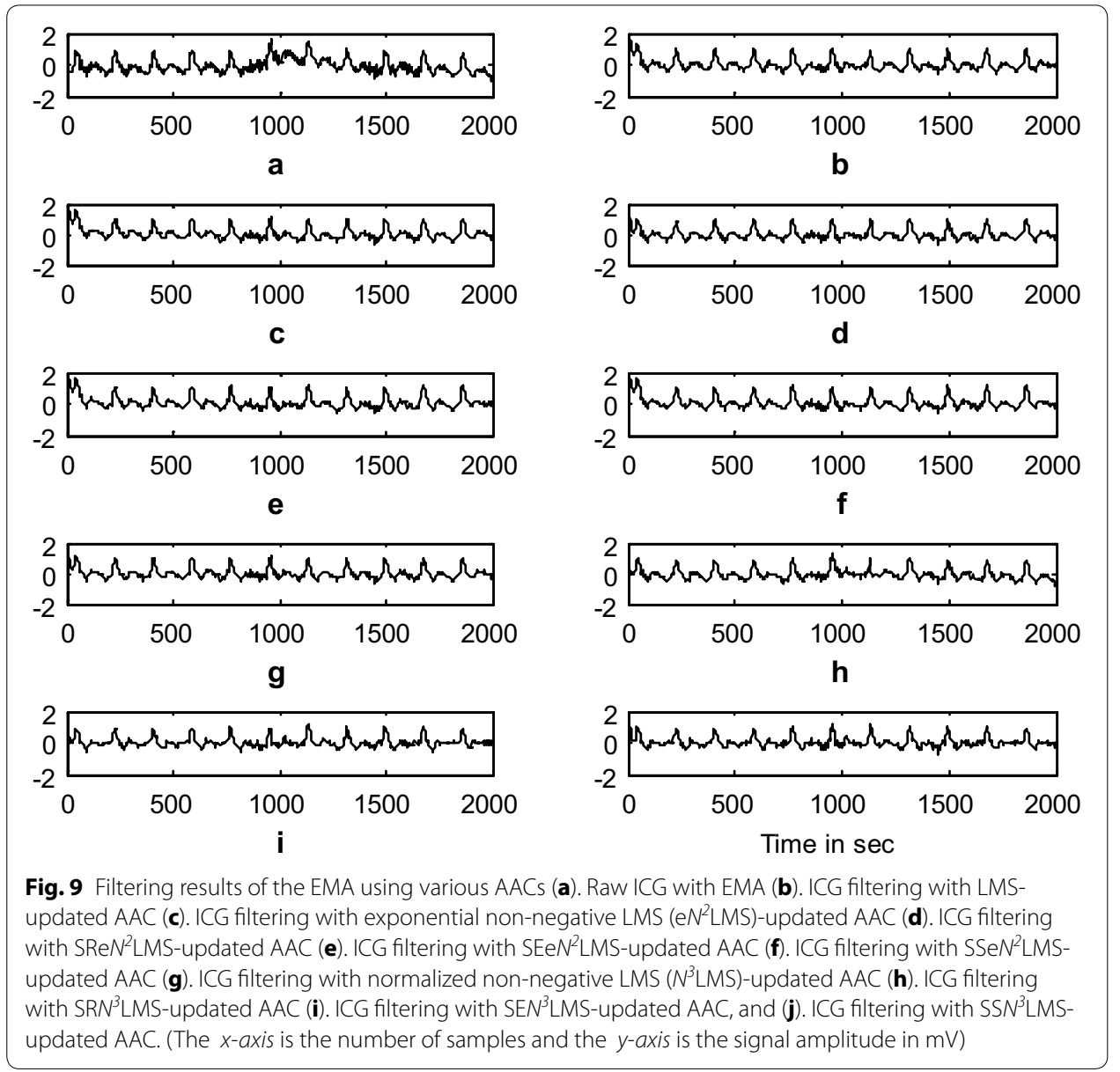




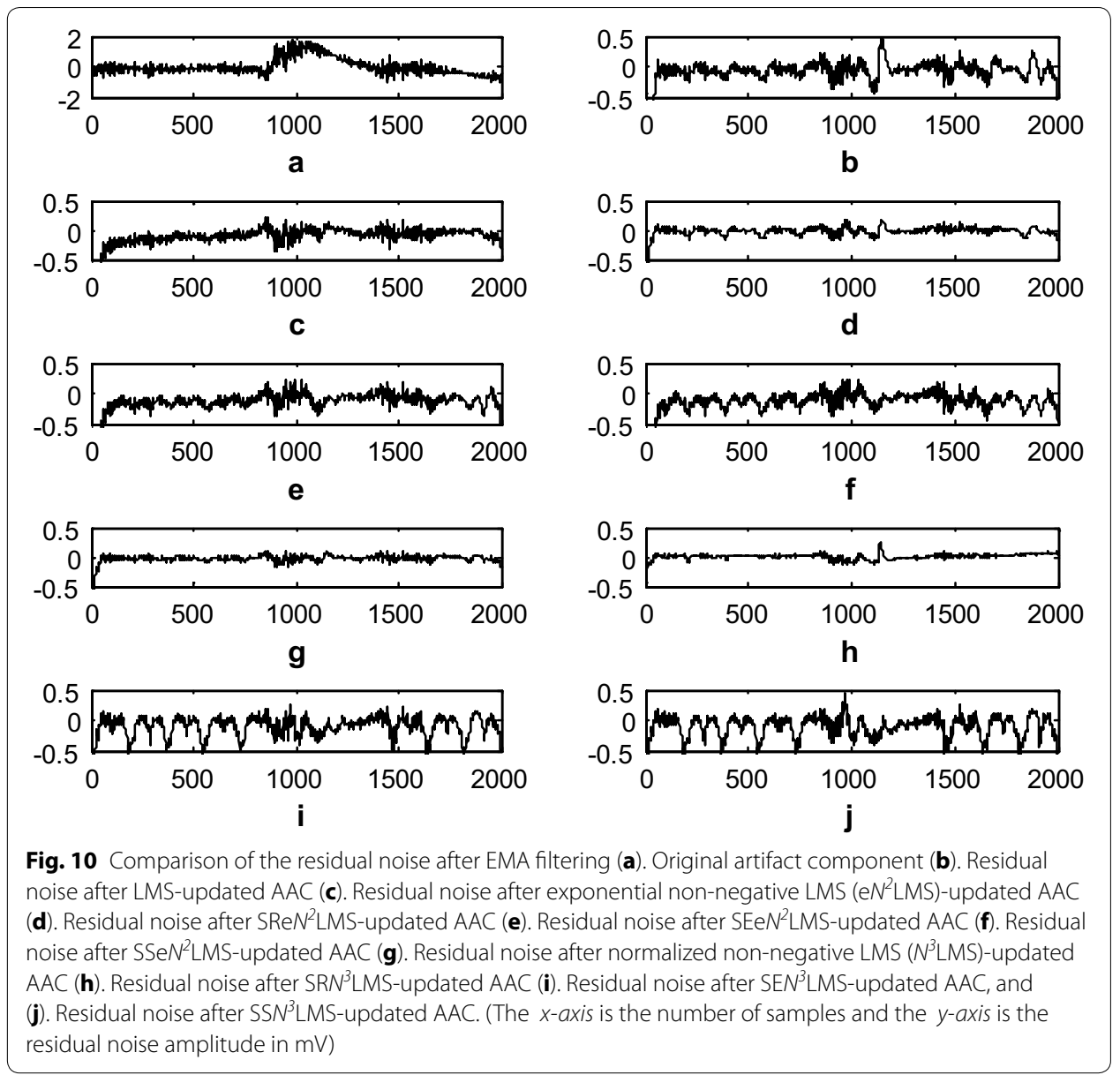

various algorithms. The data in Fig. 12h show that the residual error in the case of $S R$ $N^{3} \mathrm{LMSis}$ less than that of the other algorithms. This also is supported by the SNR table, i.e., among all of the algorithms, $S R N^{3} \mathrm{LMS}$ achieves the highest SNR at $8.4466 \mathrm{dBs}$. The data in Table 2 also show that all of the non-negative algorithms have non-negative weight coefficients when filtering the various artifacts. Finally, all of the performance measures indicate that $S R N^{3} \mathrm{LMSis}$ better than the other algorithms with respect to SNR, RRMSE, convergence and computational complexity.

\section{Conclusion}

This paper presents a new technique for enhancing ICG signals for telecardiology applications. The primary feature of the proposed adaptive artifact canceller is that it does not require a reference signal. The proposed model itself constructs a reference signal 

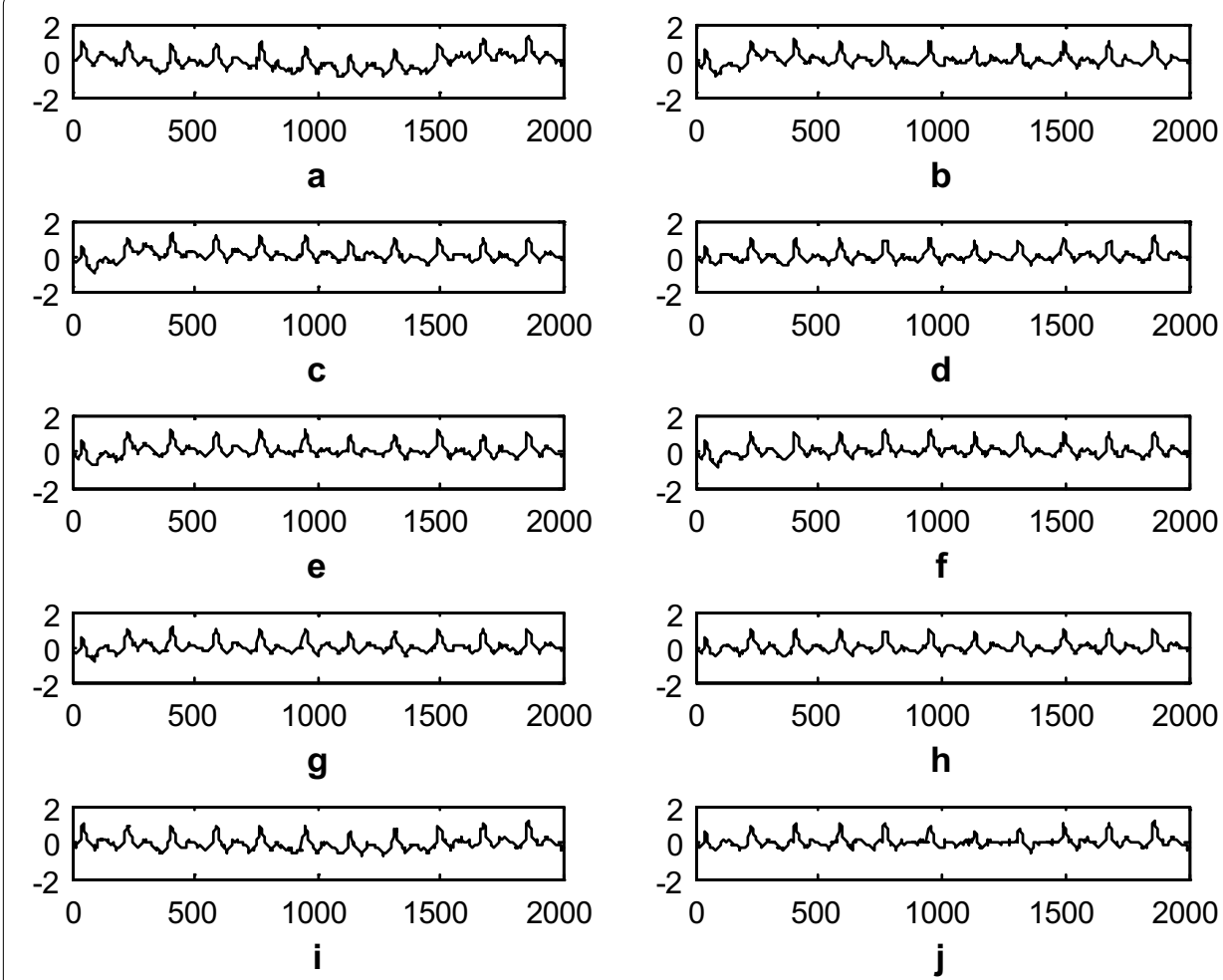

Fig. 11 Filtering results for IMA from the various AACS (a). ICG signal with IMA (b). ICG filtering with LMSupdated AAC, (c). ICG filtering with exponential non-negative LMS (eN²LMS)-updated AAC (d). ICG filtering with SReN²LMS-updated AAC (e). ICG filtering with SEeN²LMS-updated AAC (f). ICG filtering with SSeN $N^{2} L M S-$ updated AAC (g). ICG filtering with normalized non-negative LMS (N ${ }^{3}$ MS)-updated AAC (h). ICG filtering with SRN $N^{3} L M S$-updated AAC (i). ICG filtering with SEN ${ }^{3} L M S$-updated AAC, and (j). ICG filtering with SSN $N^{3}$ LMSupdated AAC. (The $x$-axis is the number of samples and the $y$-axis is the signal amplitude in $\mathrm{mV}$ )

using DWT decomposition. This constructed signal is used to train the filter weight coefficients for the noise cancelation process. To avoid computational divergence caused by negative weights during abnormal heart conditions, we use a non-negative LMS algorithm and its variants. Based on this constraint, we developed various non-negative algorithms, that is, $e N^{2} L M S, S R e N^{2} L M S, S E e N^{2} L M S$, SS $e N^{2} L M S, N^{3} L M S, S R N^{3} L M S$, $S E N^{3} L M S$ and $S S N^{3} L M S$. Among these algorithms, the sign regressor-based algorithms require fewer multiplications and achieve better convergence because of an additional normalization factor used in the signum function. Additionally, the convergence characteristics of the sign regressor version are slightly inferior to its unsigned version. Therefore, with respect to the SNR shown in Table 1, the weight coefficients shown in Table 2, the filtering results, the residual error, the RRMSE curves and the computational 


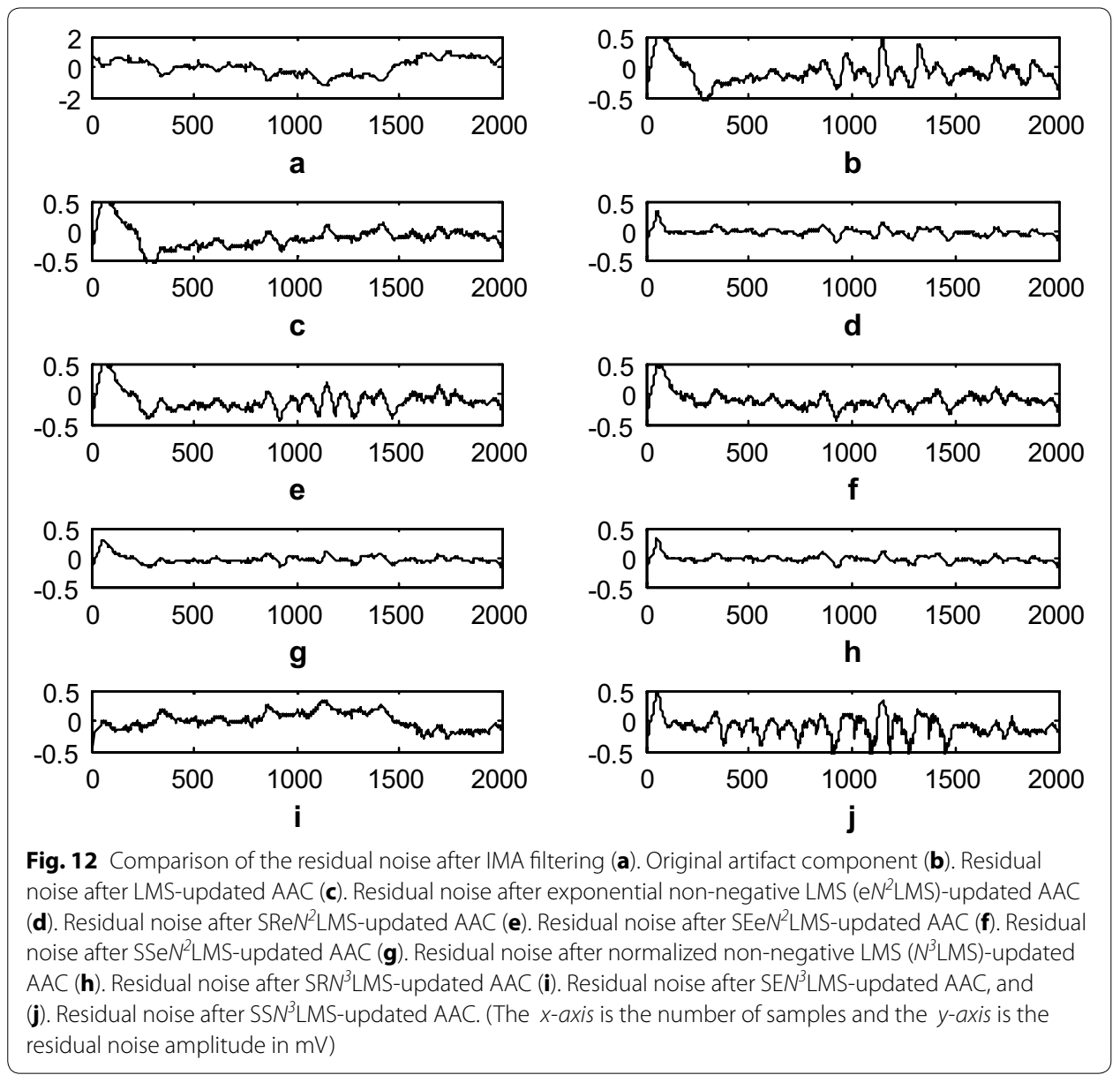

complexity, the results show that the $S R N^{3} L M S$-based AAC performs better than the other algorithms. Hence, this DWT-based AAC is well-suited for noise cancelation in remote health care monitoring systems.

\section{Authors' contributions}

MM involved in the ICG data collection, Pre-processing. KCBR explained the algorithms and methodology. Then MM prepared MATLAB coding for these concepts. KCBR also corrected the syntax and grammar mistakes in the manuscript. Both authors read and approved the final manuscript.

\section{Author details}

1 Department of Electronics and Communication Engineering, Jawaharlal Nehru Technological University, Kakinada, AP 533003, India. ${ }^{2}$ Department of Electronics and Communication Engineering, JNTUK, University College of Engineering, Vizianagaram, AP 535002, India.

\section{Acknowledgements}

The authors would like to thank the Psychophysiology Group, Department of Psychology, The University of Sydney, Sydney, Australia for providing raw ICG data.

\section{Competing interests}

The authors declare that they have no competing interests.

\section{Consent to publish}

The consent to publish has been obtained from the participant to report individual's data. 


\section{References}

American Heart Association (2015) Heart disease and stroke statistics. American Heart Association

Barros AK, Yashizawa M, Yasuda Y (1995) Filtering non-correlated noise in impedance cardiography. IEEE Trans Biomed Eng 42(3):324-327

Bernstein DP (1986) Continuous noninvasive real-time monitoring of stroke volume and cardiac output by thoracic electrical bioimpedance. Crit Care Med 14(10):898-901

Brown BH, Barber DC, Morice AH, Leathard AD (1994) Cardiac and respiratory related electrical impedance changes in the human thorax. IEEE Trans Biomed Eng 41(8):729-734

Chen J, Richard C, Bermudez JCM, Honeine P (2011) Nonnegative least mean square algorithm. IEEE Trans Signal Process 59(11):5225-5235

Chen J, Richard C, Bermudez JCM, Honeine P (2014a) Variants of non negative least mean square algorithm and convergence analysis. IEEE Trans Signal Process 62(15):3990-4005

Chen J, Bermudez JCM, Richard C (2014b) Steady state performance of non negative least mean square algorithm and its variants. IEEE Signal Process Lett 21(8):928-932

Coifman RR, Donoho DL (1995) Translation invariant de-noising. In: Antoniadis A, Oppenheim G (eds) Wavelets and statistics. Springer, New York, pp 125-150

Dromer O, Alata O, Bernard O (2009) Impedance cardiography filtering using scale fourier linear combiner based on RLS algorithm. In: 31st annual international conference of the IEEE EMBS Minneapolis, Minnesota, USA, pp 6930-6933, 2-6 September, 2009. ISBN: 978-1-4244-3296-7/09

Eweda E (1990) Analysis and design of a signed regressor LMS algorithm for stationary and nonstationary adaptive filtering with correlated Gaussian data. IEEE Trans Circuits Syst 37(11):1367-1374

Farhang-Boroujeny B (1998) Adaptive filters-theory and applications. Willey, Chichester

Goedhart AD, Kupper N, Willemsen G, Boomsma DI, De Geus EJ (2006) Temporal stability of ambulatory stroke volume and cardiac output measured by impedance cardiography. Biol Psychol 72(1):110-117

Ishiguro T, Umezu A, Yasuda Y, Horihata S, Barros AK (2006) Modified scaled Fourier linear combiner in thoracic impedance cardiography. Comput Biol Med 36:997-1013

Javaid AQ, Wiens AD, Fesmire NF, Weitnauer MA, Inan OT (2015) Quantifying and reducing posture-dependent distortion in ballistocardiogram measurements. IEEE J of Biomed Health Inform 19(5):1549-1556

Karthik GVS, Fathima SY, Rahman MZU, Ahamed SR, Lay-Ekuakille A (2013) Efficient signal conditioning techniques for brain activity in remote health monitoring network. IEEE Sens J 13(9):3276-3283

Koike Shinichi (1999) Analysis of adaptive filters using normalized signed regressor LMS algorithm. IEEE Trans Signal Process 47(10):2710-2723

Kubicek WG, Karnegis JN, Patterson RP, Witsoe DA, Mattson RH (1966) Development and evaluation of an impedance cardiography output system. Aerosp Med 37:1208-1212

Kubicek WG, Kottke FJ, Ramos MU (1974) The Minnesota impedance cardiograph theory and applications. Biomed Eng 9:410-416

Muzi M, Ebert TJ, Tristani FE, Jeutter DC, Barney JA, Smith JJ (1985) Determination of cardiac output using ensembleaveraged impedance cardiograms. J Appl Physiol 58:200-205

Nagel JH, Shyu LY, Reddy SP, Hurwitz BE, MCCabe PM, Schneiderman N (1989) New signal processing techniques for improved precession of non invasive impedance cardiography. Ann Biomed Eng 17:517-534

Peng H, Hu B, Shi Q, Ratcliffe M, Zhao Q, Qi Y, Gao G (2013) Removal of ocular artifacts in EEG an improved approach combining DWT and anc for portable applications. IEEE J Biomed Health Inf 17(3):600606

Percival DB, Walden AT (2000) Wavelet methods for time series analysis. Cambridge University Press, Cambridge

Rahman MZU, Ahamed SR, Reddy DVRK (2011) Efficient sign based normalized adaptive filtering techniques for cancelation of artifacts in ECG signals: application to wireless biotelemetry. Signal Process 91:225-239

Rahman MZU, Ahamed SR, Reddy DVRK (2012) Efficient and simplified adaptive noise cancellers for ECG sensor based remote health monitoring. IEEE Sens J 12(3):566-573

Rahman MZU, Karthik GVS, Fathima SY, Lay- Ekuakille A (2013) An efficient cardiac signal enhancement using time-frequency realization of leaky adaptive noise cancelers for remote heath monitoring systems. Measurement 43:3815-3835

Riese H, Groot PFC, Van Den Berg M, Kupper NHM, Magnee EHB, Rohaan EJ (2003) Large-scale ensemble averaging of ambulatory impedance cardiograms. Behav Res Methods Instrum Comput 35(3):467-477

Scherhag A, Kaden JJ, Kentschke E, SueselbeckT, Borggrefe M (2005) Comparison of impedance cardiography and thermodilution derived measurement of stroke volume and cardiac output at rest and during exercise testing. Cardiovasc Drugs Therapy 19:141-147

Sebastian T, Pandey PC, Naidu SMM, Pandey VK (2011) Wavelet Based denoising for suppression of respiratory and motion artifacts in impedance cardiography. Computing in cardiology 2011, Hangzhou, China, INSPEC Accession Number 12591921, pp 501-504, 18-21 September 2011

Sramek BB (1983) Electrical bioimpedance. Med Electron 14:95-103

Thakor NV, Zhu Y-S (1991) Applications of adaptive filtering to ECG analysis: noise cancelation and arrhythmia detection. IEEE Trans Biomed Eng 38(8):785-794

Wang X, Sun HH, Water JMVD (1995) An advanced signal processing technique for impedance cardiography. IEEE Trans Biomed Eng 42(2):224-230

Willemsen GHM, De Geus EJ, Klaver CHAM, Van Doormen LP, Carroll D (1996) Ambulatory monitoring of the impedance cardiogram. Psychophysiology 33:184-193

Woltjer HH, Bogaard HJ, de Vries PMJM (1997) The technique of impedance cardiography. Eur Heart J 18(9):1396-1403

World Health Organization (2015a) Health in 2015: from Millennium development goals to sustainable development goals. World Health Organization

World Health Organization (2015b) World Health Organization Fact Sheets on Cardiovascular Diseases. World Health Organization, Fact sheet N317, January, 2015

Yamamoto Y, Mokushi K, Tamura S, Mutoh Y, Miyashita M, Hamamoto H (1988) Design and implementation of a digital filter for beat by-beat impedance cardiography. IEEE Trans Biomed Eng 35(12):1086-1090 